\title{
Differences in presentation, severity and management of DKA in type 1 and type 2 diabetes during the COVID-19 pandemic
}

\author{
Authors: Lucy Wallett, ${ }^{\mathrm{A}}$ Punith Kempegowda, ${ }^{\mathrm{B}}$ Eka Melson, ${ }^{\mathrm{B}}$ Agata Juszczak, ${ }^{\mathrm{A}}$ Lucretia Thomas, ${ }^{\mathrm{C}}$ Dengyi Zhou, ${ }^{\mathrm{C}}$ \\ Catherine Holmes, ${ }^{A}$ Muhammad Ali Karamat, ${ }^{A}$ Sandip Ghosh, ${ }^{A}$ Wasim Hanif, ${ }^{A}$ Agnes Johnson, ${ }^{C}$ Parth Narendran ${ }^{B}$ \\ and Srikanth Bellary
}

\section{Introduction}

COVID-19 infection in people with diabetes is associated with a disproportionately increased risk of complications and mortality. ${ }^{1}$ Diabetic ketoacidosis (DKA) is an acute complication of diabetes. ${ }^{2,3}$ Little is known about DKA in the presence of COVID-19 infection. This study aimed to explore the effects of COVID-19 infection on presentation, clinical course and outcome in patients presenting with DKA.

\section{Methods}

This retrospective cohort study included all patients treated for DKA between 1 March and 30 May 2020 at University Hospitals Birmingham. Patients were categorised as COVID-positive or negative based on PCR swab test. A pre-COVID group was established as external control. Data regarding demographics, diabetes type, admission $\mathrm{pH}$, bicarbonate, lactate, glucose, serum electrolytes, urea, creatinine, time to resolution of acidosis and ketosis, complications and outcome were collected.

DKA onset was defined as the presence of hyperglycaemia (serum glucose $>11 \mathrm{mmol} / \mathrm{L}$ ), ketosis (serum ketones $>3 \mathrm{mmol} / \mathrm{L}$ or urine ketone $>+++$ ) and metabolic acidosis $(\mathrm{pH}<7.3$ or bicarbonate $<15 \mathrm{mmol} / \mathrm{L}$ ) and DKA resolution as (ketone $<0.6 \mathrm{mmol} / \mathrm{L}$ or urinary ketones $\leq++$ and $\mathrm{pH}>7.3$ or bicarbonate $>15 \mathrm{mmol} / \mathrm{L}$ ) as per national guidelines in the UK. ${ }^{4}$

\section{Results}

A total of 88 episodes were included in the final analysis (20 COVID-positive, 31 COVID-negative, 37 pre-COVID).

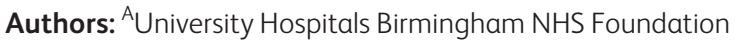

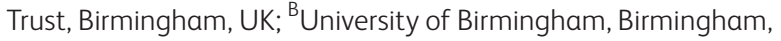
UK, and University Hospitals Birmingham NHS Foundation Trust, Birmingham, UK; ' University of Birmingham, Birmingham, UK; ' University Hospitals Birmingham NHS Foundation Trust, Birmingham, UK, and Aston University, Birmingham, UK
These were further sub-classified into type 1 (five COVID-positive, two COVID-negative, 29 pre-COVID) and type 2 diabetes (15 COVID-positive, two COVID-negative, eight pre-COVID). There was no significant difference in the severity of DKA at presentation (median for COVID positive, COVID negative and pre-COVID groups): $\mathrm{pH}$ (7.15 vs 7.2 vs 7.2 ), bicarbonate (11.4 $\mathrm{mmol} / \mathrm{L}$ vs $11 \mathrm{mmol} / \mathrm{L}$ vs $13.3 \mathrm{mmol} / \mathrm{L})$, glucose $(25.85$ $\mathrm{mmol} / \mathrm{L}$ vs $30.9 \mathrm{mmol} / \mathrm{L}$ vs $29.1 \mathrm{mmol} / \mathrm{L})$, lactate $(2.7 \mathrm{mmol} / \mathrm{L}$ vs $3.2 \mathrm{mmol} / \mathrm{L}$ vs $2.8 \mathrm{mmol} / \mathrm{L})$, serum osmolality $(314.6 \mathrm{mmol} / \mathrm{L}$ vs $323.1 \mathrm{mmol} / \mathrm{L}$ vs $316.2 \mathrm{mmol} / \mathrm{L})$. There was also no significant difference between the groups for the duration of DKA (from the time of admission to resolution of DKA: 12.5 hours vs 14.9 hours vs 17.9 hours for COVID-positive, COVID-negative and pre-COVID groups, respectively; Fig 1). COVID-positive patients with type 1 diabetes were more hyperglycaemic on admission compared to COVID-negative and pre-COVID patients. There was a significantly higher incidence of type 2 diabetes in COVID-positive patients than in pre-COVID or COVID-negative groups. COVID-negative patients had more episodes of hypokalaemia and hyperkalaemia during DKA compared to other two groups. There was no significant difference between the number of episodes of hypokalaemia in the COVID-positive compared to the preCOVID group. In patients with type 2 diabetes, hypokalaemia was more common in COVID-negative patients.

\section{Conclusion}

COVID infection appears to influence the natural history of DKA differently in type 1 and type 2 diabetes. Infection was associated with increased hyperglycaemia in type I diabetes and greater acidosis on presentation with DKA. People with type 2 diabetes were presenting unusually in DKA when infected with COVID. These patients also had higher mortality rates. There is need for a multi-centre approach to conduct larger cohort studies in this area.

\section{Conflicts of interest}

None declared. 

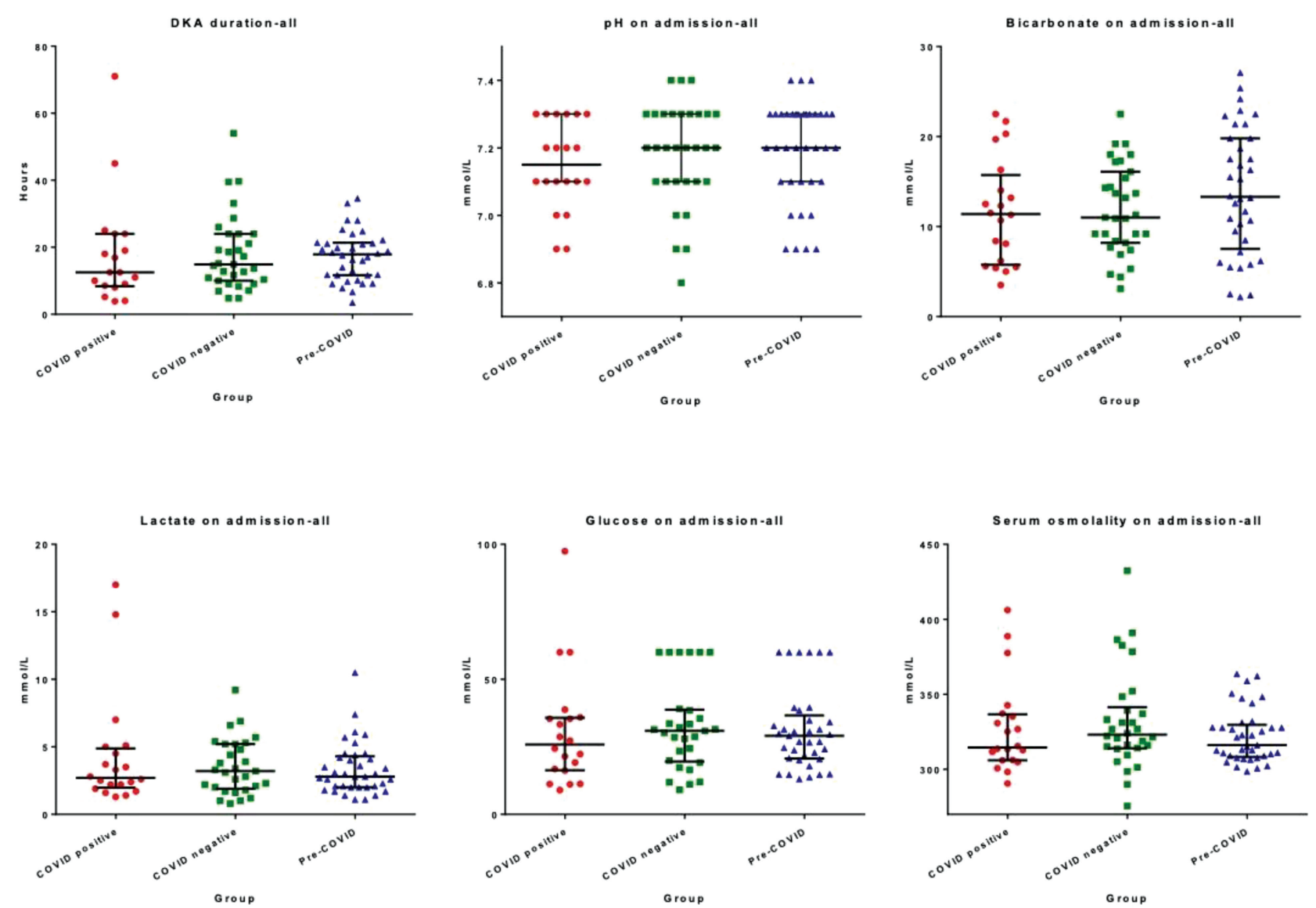

Fig 1. Differences in DKA duration, acidity (measured in $\mathrm{pH}$ and bicarbonate), glucose, lactate and serum osmolality at admission between COVID-positive, COVID-negative and pre-COVID groups with any type of diabetes.

\section{References}

1 Kumar A, Arora A, Sharma P et al. Is diabetes mellitus associated with mortality and severity of COVID-19? A meta-analysis. Diabetes Metab Syndr Clin Res Rev 2020;14:535-45.

2 Savage MW, Dhatariya KK, Kilvert A et al. Joint British Diabetes Societies guideline for the management of diabetic ketoacidosis. Diabet Med 2011;28:508-15.
3 Braatvedt G, Kwan A, Dransfield W et al. Differing protocols of managing adult diabetic ketoacidosis outside of the intensive care unit make no difference to the rate of resolution of hyperglycaemia and acidosis. N Z Med J 2019;132:13-23.

4 Gupta R, Hussain A, Misra A. Diabetes and COVID-19: evidence, current status and unanswered research questions. Eur J Clin Nutr 2020;74:864-70. 